UCRL-JC-118715

PREPRINT

\title{
Livermore Imaging Fourier Transform Infrared Spectrometer (LIFTIRS)
}

\author{
M. Carter \\ C. Bennett \\ D. Fields \\ F. Lee
}

This paper was prepared for submittal to the SPIE Conference, Orlando, Florida

April 17-21, 1995

May, 10, 1995

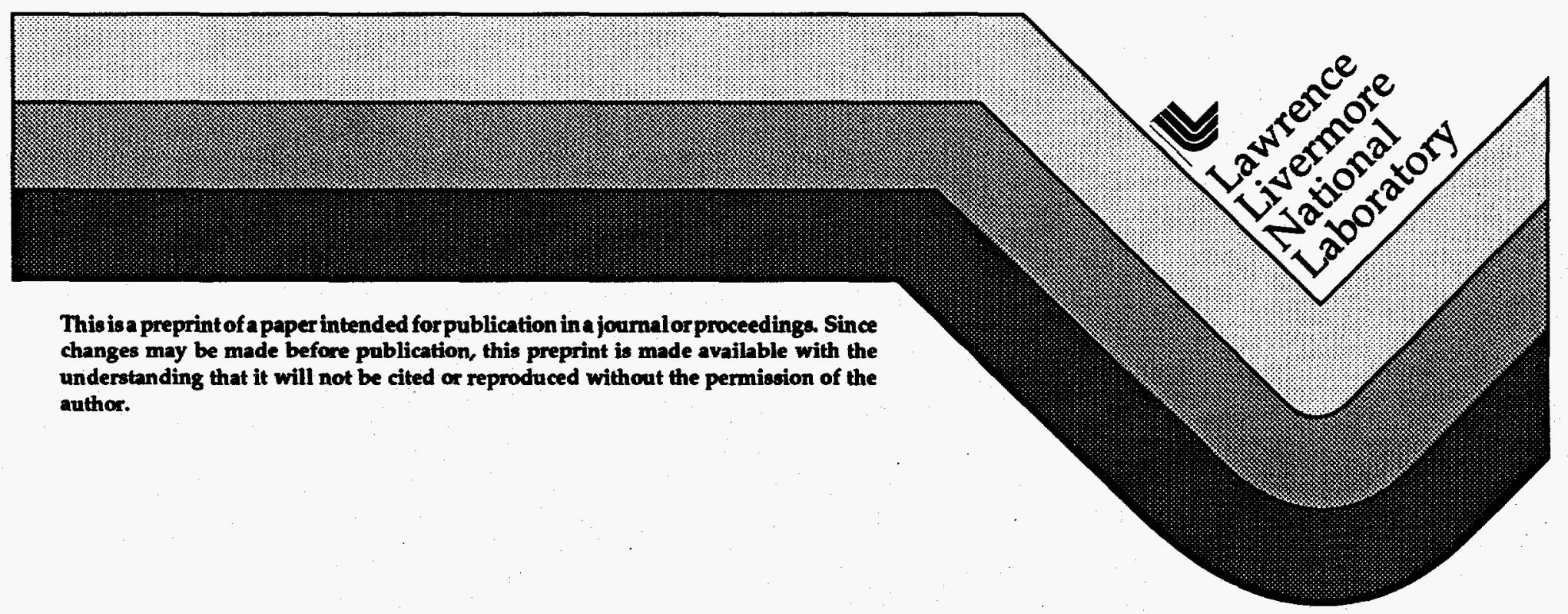

RECEIVED

DISTRIBUTION OF THIS DOCUMENT IS UNLIMTTED

AUG 241995

$0 \mathrm{TH}$ 


\section{DISCLAIMER}

This document was prepared as an account of work sponsored by an agency of the United States Government. Neither the United States Government nor the University of California nor any of their employees, makes any warranty, express or implied, or assumes any legal liability or responsibility for the accuracy, completeness, or usefulness of any information, apparatus, product, or process disclosed, or represents that its use would not infringe privately owned rights. Reference herein to any specific commercial product, process, or service by trade name, trademark, manufacturer, or otherwise, does not necessarily constitute or imply its endorsement, recommendation, or favoring by the United States Government or the University of California. The views and opinions of authors expressed herein do not necessarily state or reflect those of the United States Government or the University of California, and shall not be used for advertising or product endorsement purposes. 


\section{DISCLAIMER}

Portions of this document may be illegible in electronic image products. Images are produced from the best available original document. 


\title{
Livermore Imaging Fourier Transform Infrared Spectrometer
} (LIFTIRS)

\author{
Michael R. Carter, Charles L. Bennett, David J. Fields and F. Dean Lee \\ Lawrence Livermore National Laboratory \\ P.O. Box 808, Livermore, CA. 94551
}

\begin{abstract}
Lawrence Livermore National Laboratory is currently operating a hyperspectral imager, the Livermore Imaging Fourier Transform Infrared Spectrometer (LIFTIRS). This instrument is capable of operating throughout the infrared spectrum from 3 to $12.5 \mu \mathrm{m}$ with controllable spectral resolution. In this presentation we report on it's operating characteristics, current capabilities, data throughput and calibration issues.
\end{abstract}

Keywords: hyperspectral imaging, remote sensing, Fourier transform spectrometry

\section{INTRODUCTION}

The LIFTIRS instrument is being developed for a wide range of infrared remote sensing applications including the identification and mapping of gaseous effluents, the remote measurement of surface temperatures and the remote classification of surface material types based on their emissivity in the infrared. We have chosen an imaging Fourier transform spectrometer (IFTS) for these applications for several reasons. The primary advantages of the IFTS are the capacity to acquire more than an order of magnitude more spectral channels than alternative systems with more than an order of magnitude greater étendue than for alternative systems. The primary disadvantage of Fourier transform spectroscopy is that, for a given spatial location, all spectral channels are not recorded simultaneously. This results in sensitivity to temporal fluctuations, either random or periodic. Fourier transform instruments also have great operational flexibility particularly in their variable spectral resolution.

LIFTIRS, like all imaging spectrometers, acquires a three dimensional "data cube" consisting of 2 spatial and 1 spectral dimension. LIFTIRS acquires a full 2 dimensional image per frame, with successive frames associated with different positions of a moving mirror in a Michelson interferometer. This is illustrated in figure 1, where a set of frames of a complete 2 dimensional scene are shown in a stack. The variation of light intensity for a single pixel in the scene as a function of the position of the moving mirror is indicated by the interferogram curve in figure 1. Fourier transformations of the interferograms for each pixel in the field of view then produce the spectral dimension of the data cube, as is illustrated in figure 1.

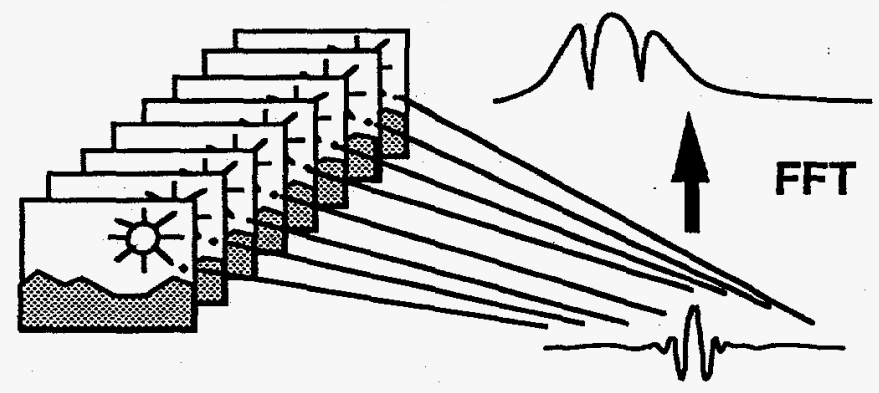

Figure 1. A raw data cube for an imaging Fourier transform spectrometer where an interferogram is acquired for each pixel in a scene. These interferograms can be Fourier transformed to produce a Hyperspectral data cube. 
A more complete discussion of the advantages and disadvantages of imaging Fourier transform spectrometers compared to dispersive techniques was presented at the 1993 SPIE conference in Orlando, Florida1. Early measurement results from the LIFTIRS device were presented at the SPIE Substance Identification Conference in 1993 in Innsbruck, Austria ${ }^{2}$. Recent measurements of chemical vapor plumes with a much improved device are presented by Bennett, et al. at this conference ${ }^{3}$.

\section{BASIC PRINCIPLES OF IFTS}

LIFTIRS is based on the Michelson interferometer, with one fixed mirror and one moving mirror. The light transmitted through the interferometer is measured as a function of the displacement of the moving mirror from the zero phase difference position. The Fourier transform of this "interferogram" yields the spectrum. An imaging spectrometer is obtained by "looking through" the interferometer with a camera, and constructing the Fourier transform of the variations in light intensity at each pixel as a function of the position of the moving mirror. The optical layout of an IFTS is sketched in figure 2 .

Typical rays emerging from two representative points are drawn. One point is located on the optical axis. The second point is displaced by a distance $y$ from the optical axis. The object plane is located at a distance equal to the focal length $f$ of the input collimating lens. The object plane may be a real emitting surface, or may be the focal plane of a telescope or microscope. The beam splitter transmits a fraction $T$ of the incident light and reflects a fraction $\mathrm{R}$. These coefficients in general depend on the polarization of the incident light, the angle $\theta$, and the wavelength.

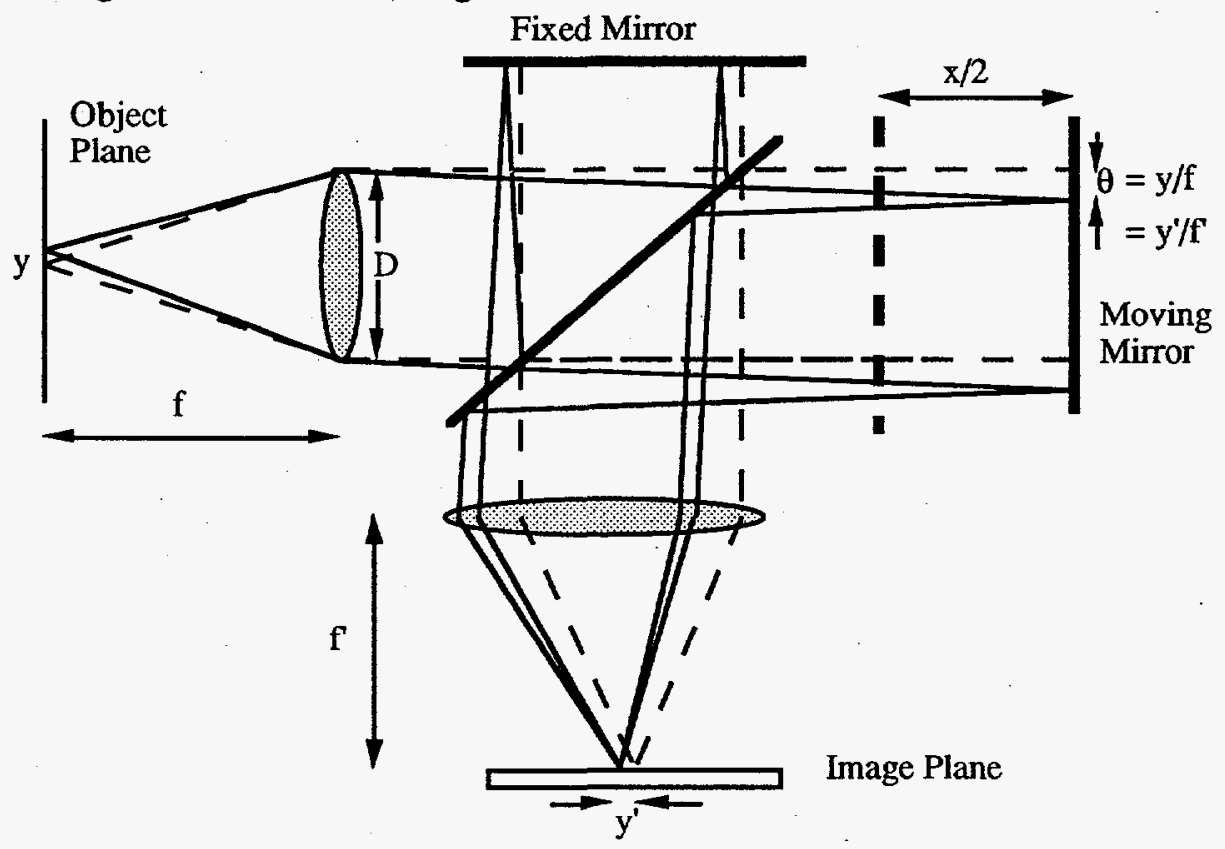

Figure 2. A sketch of the optics of an Imaging Fourier Transform Spectrometer

\section{SENSOR OPERATIONS}

LIFTIRS can be operated in either the $8-12 \mu \mathrm{m}$ or the $3-5 \mu \mathrm{m}$ region of the spectrum depending on the choice of infrared focal plane arrays. We are currently operating a Ga:Si 128 x 128 FPA for our 8-12 
$\mu \mathrm{m}$ data collections and a $256 \times 256$ InSb FPA for our 3-5 $\mu \mathrm{m}$ operations. Both focal planes were purchased from AMBER Engineering. The Ga:Si array can be read out at rates up to 1000 frames per second. The InSb FPA is limited to 125 frames per second. The data is acquired using a multichannel, 12 bit, data acquisition system. The data is stored, real-time, in a large scale memory (512 MBytes). Typical operating parameters for the 3-5 $\mu \mathrm{m}$ and 8-12 $\mu \mathrm{m}$ operations are shown in table 1 .

\begin{tabular}{|lll|}
\hline Parameter & LWIR & MWIR \\
\hline Detector & Ga:Si & InSb \\
Spectral range & $8-12.5 \mu \mathrm{m}$ & $3.3-4.9 \mu \mathrm{m}$ \\
Image format & $128 \times 128$ & $256 \times 256$ \\
pixel size & $50 \mu \mathrm{m}$ & $30 \mu \mathrm{m}$ \\
IFOV & $0.55 \mathrm{mrad}$ & $0.35 \mathrm{mrad}$ \\
Qe & $4 \%$ & $92 \%$ \\
Beam Splitter & $\mathrm{KBr} / \mathrm{Ge}$ & $\mathrm{KBr} / \mathrm{Ge}$ \\
frame rate (maximum) & $1000 \mathrm{~Hz}$ & $125 \mathrm{~Hz}$ \\
spectral resolution & variable to $0.25 \mathrm{~cm}^{-1}$ & variable to $0.25 \mathrm{~cm}^{-1}$ \\
\hline
\end{tabular}

Table 1. Operating parameters for the MW and LW LIFTIRS device.

The basic operation of LIFTIRS involves collection of a series of infrared images at different moving mirror locations. The resulting "data cube" is a collection of interferograms for each spatial pixel in the image. Each interferogram is then Fourier transformed to produce a spectral data cube. The spectral resolution of the data cube is determined by the total distance of travel of the moving mirror from the zero phase difference position. For a two sided interferogram consisting of $\mathrm{N}$ points, the corresponding spectrum will contain $\mathrm{N} / 2$ spectral channels equally spaced extending from 0 up to the Nyquist sampling limit, $k_{\max }=1 /(2 \Delta x)$, where $\Delta x$ is the sampling interval. Figure 3 shows a typical interferogram for a single pixel in the LWIR LIFTIRS device. These interferograms are phase corrected, apodized and Fourier transformed resulting in an intensity vs. wavenumber, $k$. The FFT of the interferogram is also shown in figure 3.
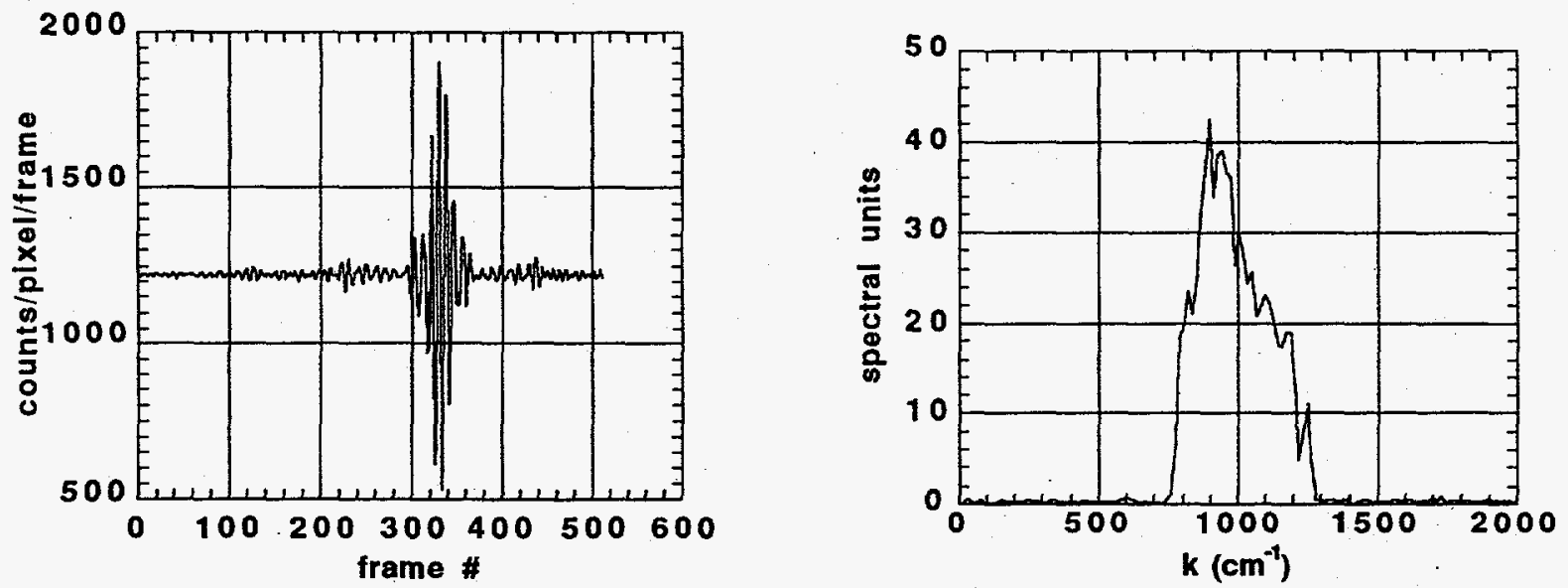

Figure 3. The time history of the intensity in one pixel vs. frame number (or mirror position) is shown on the left. The result of the FFT of the interferogram is shown on the left vs. wavenumber. 
The total data acquisition time for a LIFTIRS data cube is determined by the number of frames to be acquired and the frame rate and thus depends on the desired spectral resolution, and the choice of $\mathrm{k}_{\max }$. Figure 4 shows typical data cube acquisition times for the MWIR and LWIR LIFTIRS operations. Both curves in figure 4 assume double sided interferograms and a $\mathrm{k}_{\max }$ of $3949.5 \mathrm{~cm}^{-1}$. The difference in acquisition time between the MWIR and the LWIR is due to the difference in frame rate of the two systems. The acquisition times below are based on typical operating parameters of 400 frames/sec for the LWIR and 100 frames/sec for the MWIR. Shorter acquisition times are possible for a given spectral resolution if one acquires single sided interferograms or if $\mathrm{k}_{\max }$ is reduced. These both will result in reduced signal to noise in the resulting spectra.

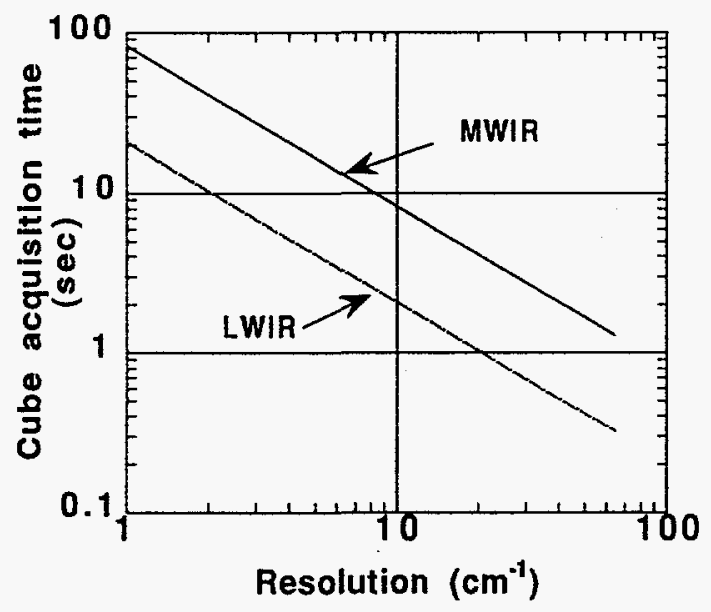

Figure 4. Data cube acquisition time for LIFTIRS MWIR and LWIR operations. Both curves assume $\mathrm{k}_{\max }=3949.5 \mathrm{~cm}^{-1}$, double sided interferograms and 100 frames $/ \mathrm{sec}$ for the MWIR and 400 frames $/ \mathrm{sec}$ for the LWIR.

\section{SENSOR CHARACTERIZATION}

The signal measured by the LIFTIRS instrument is the sum of both modulated signal from the scene of interest and background signal from sources such as emission from the instrument and dark current. Some of the background sources are modulated by the interferometer and some are not. Unmodulated sources contribute only to the DC levels measured by the detector and as such do not appear in the spectra. The unmodulated signals do, of course, contribute to the photon statistical noise level. The measured signal, $S(k, x, y)$, in the spectral domain is given by

$$
S(k, x, y)=R(k, x, y) * \phi \text { in }(k, x, y)+B(k, x, y)
$$

where $R(k, x, y)$ is the instrument responsivity, $\phi \operatorname{in}(k, x, y)$ is the incident flux and $B(k, x, y)$ is the instrumental background. The radiometric calibration consists of determining the spatial and spectral variations of both the responsivity and the instrumental background. Values for $R$ and $B$ can be determined by measuring $S(k, x, y)$ at two black bodies whose temperatures bracket the scene of interest. Assuming the incident flux, $\phi$ in $(k, x, y)$, can be calculated using Planck's law, we now have two equations and two unknowns, and can solve for $R$ and $B$, for each value of $k, x$ and $y$. These calibrations are then applied to subsequent data cubes to convert spectral units to spectral radiance $W / \mathrm{cm}^{2} / \mathrm{sr} / \mathrm{cm}^{-1}$. 
The resulting responsivity and background for one of the 65,536 pixels of the mid-wave LIFTIRS sensor are shown in figure 5. The calibrations shown in figure 5 were computed from two $64 \mathrm{~cm}^{-1}$ resolution data cubes of two black body cavities $\left(15^{\circ} \mathrm{C}\right.$ and $\left.20^{\circ} \mathrm{C}\right)$. The data cubes were each $256 \times 256$ pixels x 256 frames. The interferograms were phase corrected and boxcar apodized. Each data cube acquisition took 2.56 seconds.
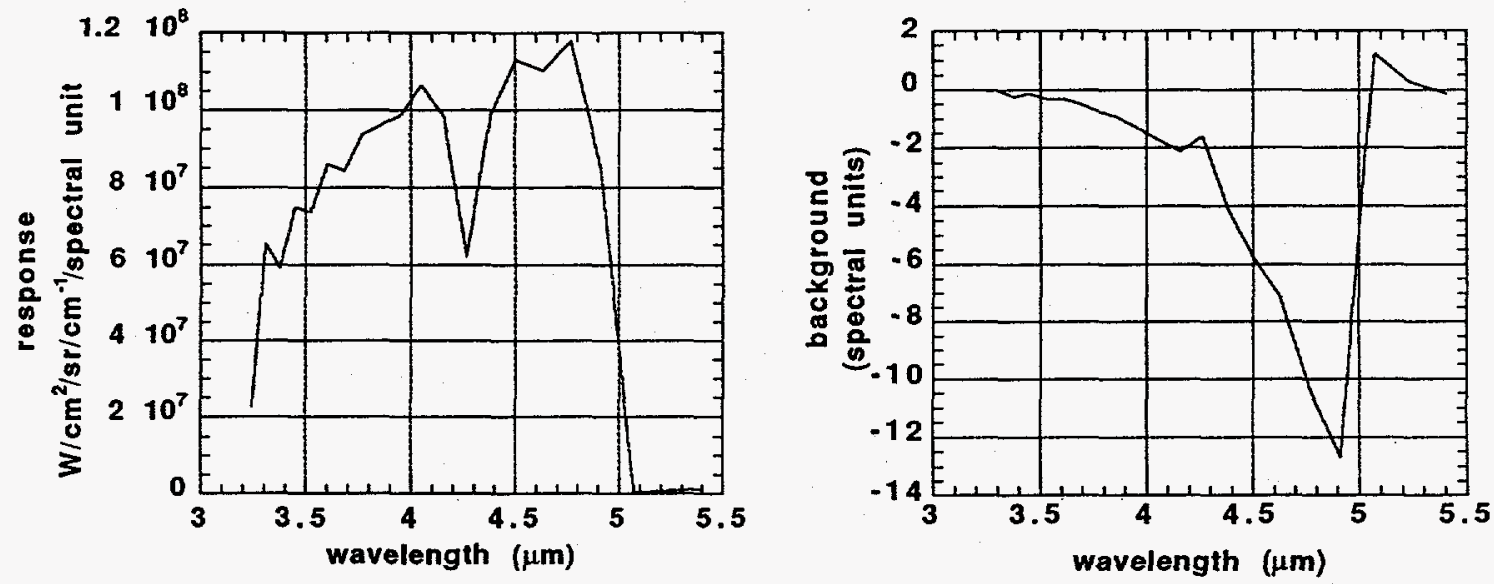

Figure 5. The measured responsivity and background for a single pixel for the MWIR LIFTIRS device shows response out to about $4.8 \mu \mathrm{m}$.

Figure 6 shows the measured SNR versus wavelength for the MWIR LIFTIRS looking at a $17.5^{\circ}$ $\mathrm{C}$ blackbody. This SNR was calculated by computing the difference in the measured/calibrated spectrum and the calculated spectrum of a $17.5^{\circ} \mathrm{C}$ blackbody. The spectral calibration coefficients $\mathrm{R}$ and $\mathrm{B}$ required to convert the measured spectrum to spectral radiance were taken from measurements of a $15^{\circ}$ and $20^{\circ} \mathrm{C}$ blackbody. This SNR includes noise both in the spectral domain and in the spatial domain. This includes any uncertainties in the spectral and spatial calibrations. All three data cubes, the two calibrations and the test data, were taken at $64 \mathrm{~cm}^{-1}$ resolution and each required a 2.5 second acquisitions. 


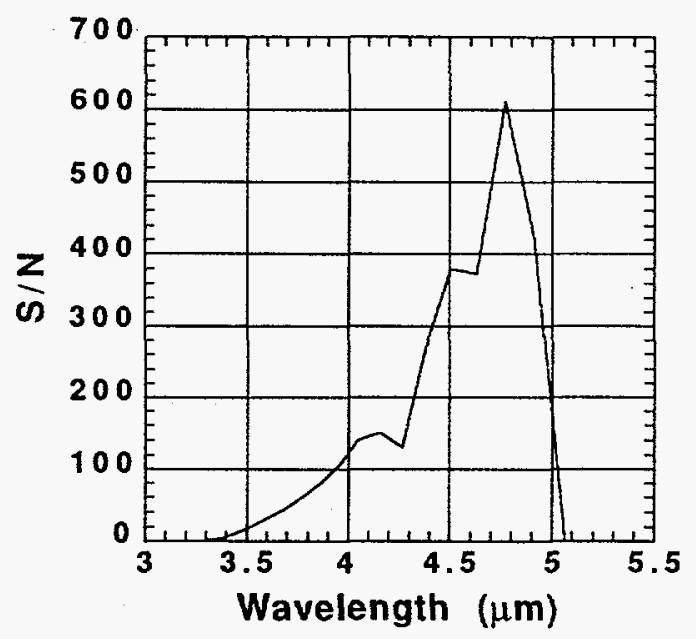

Figure 6. Measured $\mathrm{S} / \mathrm{N}$ ratio for the MWIR LIFTIRS looking at a $17.5^{\circ} \mathrm{C}$ Black Body. The spectral resolution is $64 \mathrm{~cm}^{-1}$ and the acquisition time is 2.5 seconds.

The measured responsivity and instrument background for one of the 16,384 pixels in the LWIR LIFTIRS sensor are shown in figure 7. These calibration coefficients were measured using $15^{\circ} \mathrm{C}$ and $30^{\circ} \mathrm{C}$ blackbody data cubes and again are measured for each pixel in the image. The long wave cutoff in the response is set by an IR band pass filter. These data cubes are taken at $16 \mathrm{~cm}^{-1}$ spectral resolution. Each calibration data cube was acquired in 1.28 seconds.
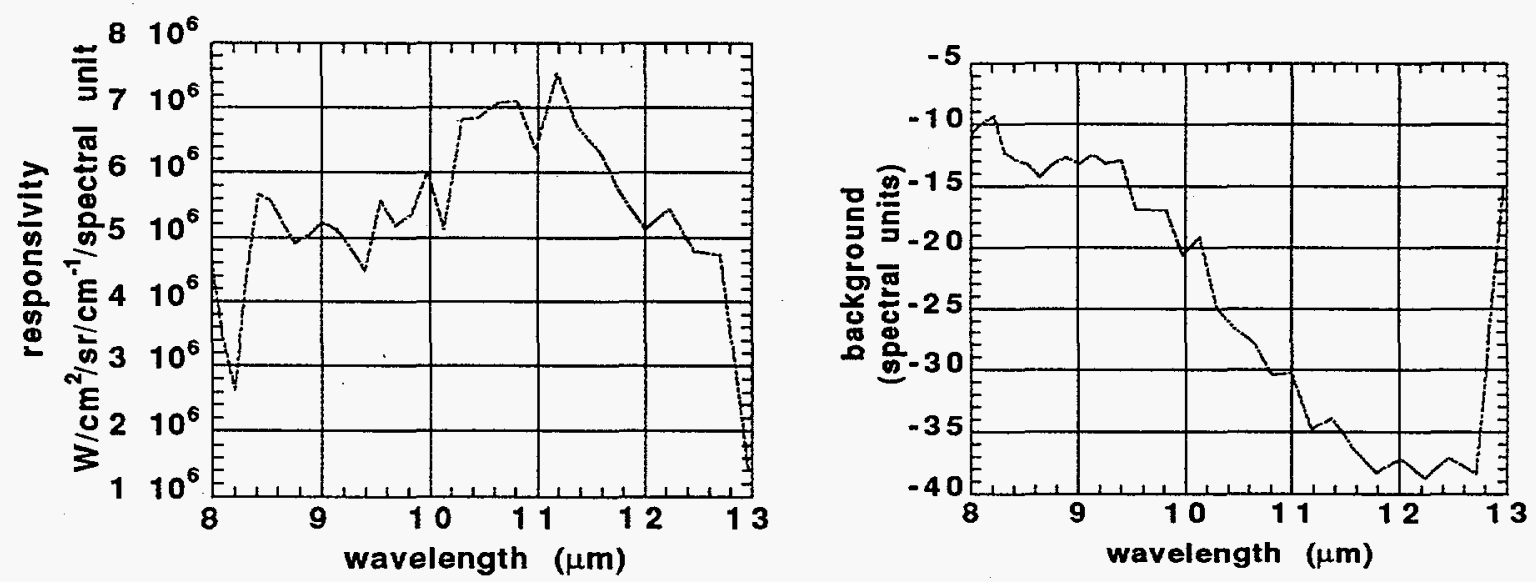

Figure 7. The measured responsivity and background for a single pixel for the LWIR LIFTIRS device shows response out to about $12.5 \mu \mathrm{m}$.

Figure 8 shows the measured SNR versus wavelength for the LWIR LIFTIRS looking at a $20^{\circ} \mathrm{C}$ blackbody. This SNR was calculated by computing the difference in the measured/calibrated spectrum and the calculated spectrum of a $20^{\circ} \mathrm{C}$ blackbody. The spectral calibration coefficients $\mathrm{R}$ and $\mathrm{B}$ required to convert the measured spectrum to spectral radiance were taken from measurements of a $15^{\circ}$ and $30^{\circ} \mathrm{C}$ 
blackbody. This SNR includes noise both in the spectral domain and in the spatial domain. This includes any uncertainties in the spectral and spatial calibrations. All three data cubes, the two calibrations and the test data, were taken at $16 \mathrm{~cm}^{-1}$ resolution and required a 1.28 second acquisition each.

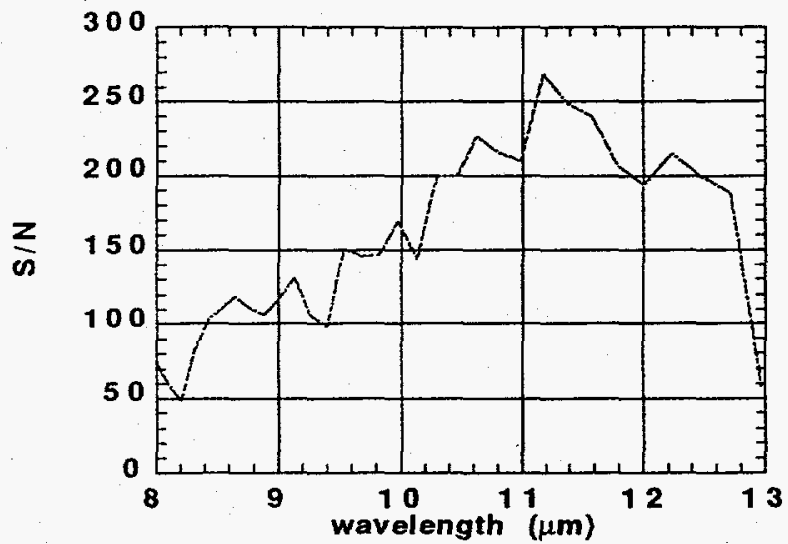

Figure 8. Measured S/N ratio for the LWIR LIFTIRS looking at a $20^{\circ} \mathrm{C}$ black body. The spectral resolution is $16 \mathrm{~cm}^{-1}$ and the acquisition time is 1.28 seconds.

\section{CONCLUSIONS}

We have developed, characterized and fielded a flexible hyperspectral imaging system based on the Michelson Fourier transform interferometer. This sensor can be operated either in the mid-wave IR (3-5 $\mu \mathrm{m})$ or the thermal IR $(8-12 \mu \mathrm{m})$ depending on the choice of infrared focal plane array. This sensor has unique operating characteristics that make it quite useful for a variety of applications including, gaseous effluent monitoring, hyperspectral measurements of temperature and emissivity of solid surfaces and infrared signature studies.

\section{ACKNOWLEDGMENTS}

This work was performed under the auspices of the U.S. Department of Energy under Contract No. W-7405-Eng-48: We thank Ray Mitchell, Tom Biesiada, Bill Aimonetti, Chuck Lee, Jonathon Marks, Ron Robinson, John Hernandez, and Noami Fortes for their help in the development, characterization and fielding of LIFTIRS. We also thank Mark Eckart for providing support for this effort.

\section{REFERENCES}

1. C. L. Bennett, M. Carter, D. Fields and J. Hernandez, "Imaging Fourier Transform Spectrometer", p. 191-200, in the proceedings of the Imaging Spectrometry of the Terrestrial Environment, April 14-15, 1993, Orlando, Fl., SPIE vol 1937.

2. M. R. Carter, C. L. Bennett, D. J. Fields and J. Hernandez, "Gaseous Effluent Monitoring and Identification using an Imaging Fourier Transform Spectrometer", p. 16-26, in the proceedings of the Substánce Detection Systems, October 5-8, 1993, Innsbruck, Austria, SPIE vol 2092.

3. C. L. Bennett, M. R. Carter, D. J. Fields and F. D. Lee, "Infrared Hyperspectral Imaging Results from Vapor Plume Experiments", in the Proceedings of Imaging Spectrometry, April 17-19, 1995, Orlando, Fl., SPIE vol 2480. 